\title{
Geometrical enhancement of the proximity effect in quantum wires with extended superconducting tunnel contacts
}

\author{
Giorgos Fagas, ${ }^{1, *}$ Grygoriy Tkachov, ${ }^{2, \dagger}$ Andreas Pfund, ${ }^{2}$ and Klaus Richter ${ }^{2}$ \\ ${ }^{1}$ Tyndall National Institute, Lee Maltings, Prospect Row, Cork, Ireland \\ ${ }^{2}$ Institut für Theoretische Physik, Universität Regensburg, 93040 Regensburg, Germany \\ (Received 13 January 2005; revised manuscript received 30 March 2005; published 24 June 2005)
}

\begin{abstract}
We study Andreev reflection in a ballistic one-dimensional channel coupled in parallel to a superconductor via a tunnel barrier of finite length $L$. The dependence of the low-energy Andreev reflection probability $R_{A}$ on $L$ reveals the existence of a characteristic length scale $\xi_{N}$ beyond which $R_{A}(L)$ is enhanced up to unity despite the low interfacial transparency. The Andreev reflection enhancement is due to the strong mixing of particle and hole states that builds up in contacts exceeding the coherence length $\xi_{N}$, leading to a small energy gap (minigap) in the density of states of the normal system. The role of the geometry of such hybrid contacts is discussed in the context of the experimental observation of zero-bias Andreev anomalies in the resistance of extended carbon nanotube/superconductor junctions in field effect transistor setups.
\end{abstract}

DOI: $10.1103 /$ PhysRevB.71.224510

PACS number(s): 74.45.+c, 74.50.+r, 73.23.Ad

\section{INTRODUCTION}

The interest in proximity-induced superconductivity in one-dimensional (1D) electron systems ${ }^{1-13}$ has recently revived in light of successful experiments on electron transport through carbon nanotubes contacted by superconductors. ${ }^{14-17}$ Despite possibly strong electron-electron interactions, ${ }^{16,18}$ in many situations transport properties of metallic single-walled carbon nanotubes can be interpreted within a ballistic model assuming two conduction bands at the Fermi level. ${ }^{19}$ Hence, superconductor/carbon-nanotube $(S / \mathrm{CN})$ junctions can to some extent be viewed as an experimentally accessible case of $1 \mathrm{D}$ ballistic proximity structures. ${ }^{15}$

As in conventional normal-metal/superconductor $(N / S)$ junctions, the extent to which the proximity effect modifies the electronic properties of carbon nanotubes strongly depends on the quality of $S / \mathrm{CN}$ interfaces. In $S / \mathrm{CN} / S$ junctions with highly nontrivial end bonding of the tubes it is possible to achieve high transparency contacts and observe induced supercurrents between the $S$ banks. ${ }^{14,16,17}$ In a more conventional field-effect transistor setup a superconductor is sputtered on top of a nanotube covering it from the ends and in this way connecting it to the leads. ${ }^{15}$ Such contacts exhibit no observable superconducting coupling, probably because of a Schottky barrier formed at the $S / \mathrm{CN}$ interfaces. Nevertheless, in this case the proximity effect manifests itself as a pronounced zero-bias dip in the low-temperature resistance to which either of the $S / C N$ interfaces contributes independently. ${ }^{15}$

The sensitivity of the zero-bias resistance anomaly to the temperature ${ }^{15,17}$ suggests that it can be attributed to the conversion of a normal current into a supercurrent via the Andreev reflection process ${ }^{20}$ during which particles with energies much smaller than the superconducting gap $\Delta$ are coherently scattered from an $S / \mathrm{CN}$ interface as Fermi sea holes back to the normal system. Under assumption of each of the $S / \mathrm{CN}$ interfaces acting independently ${ }^{15}$ and in the picture of noninteracting electrons, such an interpretation must reconcile with the well-established 1D scattering model for a single $N / S$ contact. ${ }^{21}$ However, for a point contact of average quality (between metallic and tunnel regimes) the theory of Ref. 21 predicts a zero-bias resistance peak at temperatures $T<\Delta / k_{B}$, that is exactly the opposite to the experimental findings of Refs. 15 and 17 in the same temperature regime.

Deviations of Andreev reflection physics in 1D proximity structures from the standard model of Ref. 21 have so far been ascribed to repulsive electron interactions $\mathrm{s}^{2-4,8,10}$ or disorder in the normal channel. ${ }^{13}$ In the present paper we show that clean noninteracting 1D systems can also exhibit unusual Andreev reflection properties if the contact to the superconductor is not a pointlike one. Such contacts naturally occur in field effect transistor setups due to a finite overlap between a nanotube and a superconductor coupled in parallel. In particular, in the device of Ref. 15 this overlap was as large as $1 \mu \mathrm{m}$. To demonstrate the importance of the contact geometry, we develop a scattering model for phase-coherent electron transport through a normal 1D ballistic channel, part of which is in parallel coupling to a 2D superconductor via a low-transparency barrier (Fig. 1). This model is in many aspects different from the device of Ref. 15 and it is not expected to describe all the experimental features. However, it captures the most essential, for our purposes, attribute of the S/CN contacts, namely, their extended character. Moreover, the proposed geometry may serve as a minimum model accounting for the zero-bias resistance features reported in Refs. 22-24 for extended planar contacts between ballistic 2D electron systems and superconductors, whose crosssectional structure is similar to that shown in Fig. 1.

Our numerical simulation of elastic quasiparticle scattering shows that the probability of Andreev reflection depends on the length $L$ of the contact, approaching unity as $L$ exceeds a certain length scale $\xi_{N}$ larger than the coherence length in the superconductor $\xi_{S}$. Most importantly, at zero energy high-probability Andreev reflection occurs at any finite interfacial transparency for sufficiently long contacts. This is in sharp contrast to the situation in point junctions. ${ }^{21}$ To rationalize this result we perform a numerical analysis of the quasiparticle density of states (DOS) in the region of the 

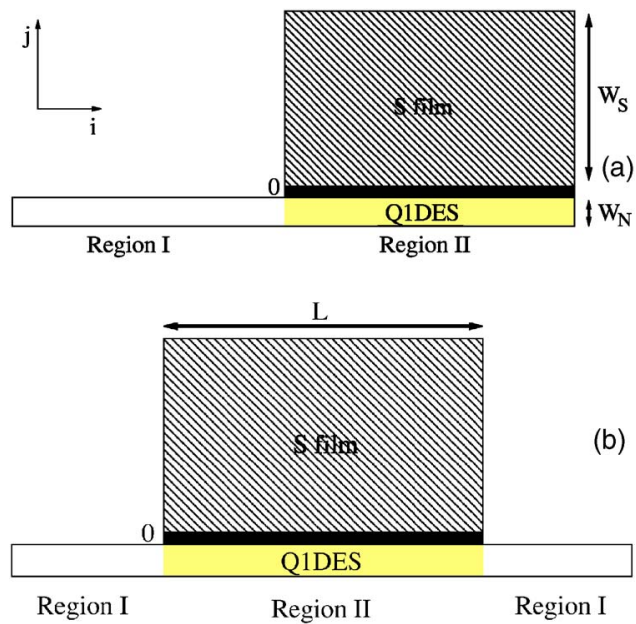

FIG. 1. Quasi-one-dimensional electron system (Q1DES) coupled to (a) semi-infinite and (b) finite length $L$ superconductor contacts. The interfacial barrier is depicted in black. The Q1DES width is chosen to be $W_{N} \approx \lambda_{F} / 2$, where $\lambda_{F}$ is the Fermi wavelength.

1D system coupled to the superconductor in the limit $L$ $\gg \xi_{N}$. The DOS is found to have a proximity-induced gap (minigap) at the Fermi level whose size $E_{g} \approx\left(\xi_{S} / \xi_{N}\right) \Delta$ is much smaller than the gap $\Delta$ in the superconductor. The minigap $E_{g}$ scales with the interfacial transparency, which implies that it is due to the formation of mixed particle-hole (Andreev) states. ${ }^{25}$ A comprehensive analysis of the energy dependence of electron scattering reveals that the gapped excitation spectrum in the proximity region results in the enhancement of the Andreev reflection probability $R_{A}(\epsilon)$ at finite (but small) energies $\epsilon<E_{g} \ll \Delta$ followed by its decrease at intermediate energies $E_{g}<\epsilon<\Delta$. At the edge of the superconducting gap $(\epsilon=\Delta)$ the dependence $R_{A}(\epsilon)$ exhibits one more peak typical for tunnel junctions. ${ }^{21}$ These features dominate the bias voltage dependence of the differential resistance which at $T \sim E_{g} / k_{B}$ displays a dip around the zero voltage similar to that observed in Ref. 15 .

Previously, zero-bias conductance anomalies have been extensively studied in mesoscopic superconducting contacts with semiconductors ${ }^{22-24,26-37}$ and metals. ${ }^{13,38-44}$ These studies have predominantly focused on the diffusive transport regime. According to the semiclassical scattering interpretation of Ref. 27, the excess conductance (i.e., exceeding the value predicted by the theory of Ref. 21) is a signature of the correlated particle-hole motion arising from multiple Andreev reflections at the interface mediated by elastic scatterers in the normal system. Even for a low-transparency contact the cumulative Andreev reflection probability can be $\sim 1$ for trajectories hitting the interface many times provided that the area of the contact is sufficiently large. In the less explored regime of ballistic propagation, a similar process, sometimes called reflectionless tunneling ${ }^{29}$, occurs in ballistic quantum wells in parallel long contacts with superconductors. In these setups, the multiple Andreev reflections are due to the back wall of the quantum well. ${ }^{22,25,35}$

Therefore, the low-bias excess conductance discussed in the present paper is a pronounced case of the reflectionless tunneling in ballistic systems where $R_{A}(\epsilon)$ can be interpreted as the cumulative Andreev reflection probability due to the correlated quantum particle-hole motion in the region of the 1D channel coupled to the superconductor. It is also known that such correlations can lead to a minigap in the quasiparticle DOS. ${ }^{25,40,41,43,45-47}$ We note that the previous studies of reflectionless transport (Refs. 27 and 35) dealt with multiple Andreev reflections semiclassically and at small energies $|\epsilon| \ll E_{g}$. Our quantum scattering approach is capable of describing the entire energy dependence of the subgap conductance which shows the crossover from reflectionless tunneling to independent electron tunneling through the barrier at the $N / S$ interface. In addition, our numerical technique allows us to tackle the realistic geometry of finite-length parallel $N / S$ contacts and to obtain an accurate complete dependence $R_{A}(L)$ which has not been studied in the previous models.

The structure of the article is as follows. In Sec. II, after a brief description of our system, we present the numerical results for the DOS in the $1 \mathrm{D}$ channel. An analytical model is also developed that helps to rationalize the low-energy regime. The length and energy dependence of the Andreev reflection probability is analyzed in Sec. III. In Sec. IV, we summarize the implications of our results with a concluding discussion on the bias voltage dependence of the resistance.

\section{TWO-GAP SPECTRAL PROPERTIES OF EXTENDED SUPERCONDUCTING TUNNEL CONTACTS}

In this section we study the density of states (DOS) in a quasi-one-dimensional electron system (Q1DES) coupled in parallel to a superconducting film via an interfacial barrier. We consider the two geometries shown in Figs. 1(a) and 1(b). The heterostructures are assumed two-dimensional and located in the plane $x, z$. Our results can be easily extended to an out-of-plane periodic structure defining a quasi-twodimensional electron system on the normal side.

\section{A. Description of the method}

To analyze the superconducting proximity effect in the Q1DES we employ a numerical approach to solve the Bogolubov-de Gennes (BdG) equation

$$
\left(\begin{array}{cc}
\hat{H} & \hat{\Delta} \\
\hat{\Delta} & -\hat{H}^{*}
\end{array}\right)\left(\begin{array}{l}
u(x, z) \\
v(x, z)
\end{array}\right)=\epsilon\left(\begin{array}{l}
u(x, z) \\
v(x, z)
\end{array}\right)
$$

for the electron $u(x, z)$ and hole $v(x, z)$ wave functions. The method allows for performing a straightforward discretization on a real-space grid of the one-particle Hamiltonian $\hat{H}$ $=-\left(\hbar^{2} / 2 m\right)\left(\partial_{x}^{2}+\partial_{z}^{2}\right)+U(x, z)-\mu$, the pairing potential $\hat{\Delta}$ $=\Delta(x, z)$, and the potential $U(x, z)$ (to be defined later). $\mu$ and $m$ are the chemical potential and the electron mass, both constant throughout the entire system. No translational invariance in the $x$ direction is invoked so that studies of the structures in Figs. 1(a) and 1(b) are possible.

The discretized $\mathrm{BdG}$ equations read 


$$
\begin{aligned}
& {[\epsilon-(4 \gamma+U(i, j)-\mu)] u(i, j)} \\
& +\gamma \sum_{i^{\prime} j^{\prime}} u\left(i^{\prime}, j^{\prime}\right)-\Delta(i, j) v(i, j)=0, \\
& {[-\epsilon-(4 \gamma+U(i, j)-\mu)] v(i, j)} \\
& \quad+\gamma \sum_{i^{\prime} j^{\prime}} v\left(i^{\prime}, j^{\prime}\right)+\Delta(i, j) u(i, j)=0,
\end{aligned}
$$

where $i$ and $j$ refer to sites on a two-dimensional lattice in the $x$ and $z$ directions, respectively, and primes denote summation over nearest neighbors. The origin of the coordinate frame is indicated in Figs. 1(a) and 1(b) by the zero. If required, Eq. (2) can be generalized to a position dependent effective mass for specific materials. ${ }^{48}$

The parameters of the numerical scheme are as follows. The potential $U(i, j)$ is infinite everywhere outside the $N$ and $S$ systems. For every $j$ within the materials, $U(i, j)=0$ for $i$ $<0$ and $U(i, j)=U_{0} \geqslant 0$ otherwise. A positive potential step $U_{0}$ accounts for the fact that the coupling to the superconductor may result in a slight reduction of the Fermi energy of the Q1DES in region (II) in Figs. 1(a) and 1(b) compared to that in the uncoupled region (I) (see Ref. 24). This turns out to be important when considering scattering of quasiparticles incident at region (II), which is analyzed in the next section. The pairing potential $\Delta(i, j)$ in Eq. (2) is assumed positionindependent and equal to $\Delta \delta_{i j}$ ( $s$ wave) in the superconductor and zero everywhere else. Although the self-consistency is ignored, the stepwise order parameter has proved to be a satisfactory approximation for studying the proximity effect in clean systems. ${ }^{29,33}$ The absolute value of $\gamma$ is inverse proportional to the mesh parameter $\alpha$, which is varied until convergence of the results is reached. A sufficient condition is $\xi_{S}, \lambda_{F} \gg \alpha$, where $\xi_{S}=\hbar v_{F} / 2 \Delta$ is the superconducting coherence length and $\lambda_{F}\left(v_{F}\right)$ is the Fermi wavelength (velocity). To simulate the effect of a relatively thick superconducting film, we consider $W_{S} / \xi_{S}=15$ for the spectral properties and $W_{S} / \xi_{S}=50$ for the results of Sec. III. Increasing this ratio does not have any quantitative effect at $|\epsilon|<\Delta$. In particular, all features discussed below are already observed for $W_{S} / \xi_{S} \approx 3$ but with prominent finite-size effects for high quasiparticle energies $|\epsilon|>\Delta$. The width of the normal region is fixed to $W_{N} / \lambda_{F} \approx 1 / 2$, allowing only one propagating mode along the Q1DES. The ratio $\xi_{S} / \lambda_{F}$ is chosen to be 2 (see also Ref. 49).

A tunneling barrier at the $N / S$ interface (dark area in Fig. 1) is introduced via an effective Hamiltonian equivalent to adding

$$
\begin{aligned}
& \sum_{i^{\prime} j^{\prime}}\left(\gamma_{N S}-\gamma\right)\left(\delta_{j, 0} \delta_{j^{\prime}, 1}+\delta_{j, 1} \delta_{j^{\prime}, 0}\right) u\left(i^{\prime}, j^{\prime}\right)=0, \\
& \sum_{i^{\prime} j^{\prime}}\left(\gamma_{N S}-\gamma\right)\left(\delta_{j, 0} \delta_{j^{\prime}, 1}+\delta_{j, 1} \delta_{j^{\prime}, 0}\right) v\left(i^{\prime}, j^{\prime}\right)=0,
\end{aligned}
$$

to Eq. (2) for every $i$ within region (II). Essentially, the above boundary conditions define the coupling between the normal and the superconducting systems via the interfacial constant $\gamma_{N S}$. The latter may arise from a formal procedure ${ }^{50}$

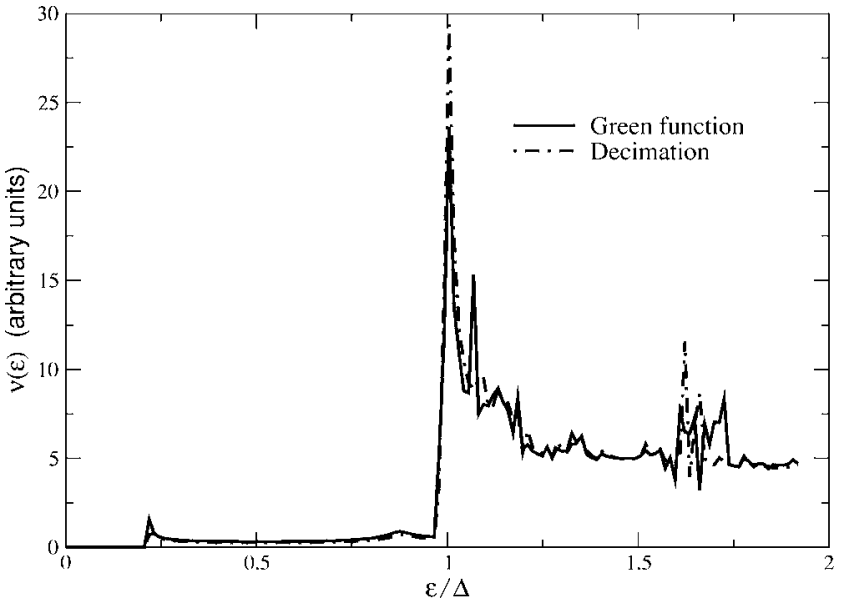

FIG. 2. DOS $\nu(\epsilon)$ of a ballistic Q1DES-superconductor system. The solid line corresponds to the semi-infinite geometry shown in Fig. 1(a) for which $\nu(\epsilon)$ is calculated directly from the Green function of the system. The dashed line corresponds to the case of a finite (but relatively long $L / \xi_{S} \gg 1$ ) $S$ film [Fig. 1(b)] where we use the decimation technique.

that projects out the degrees of freedom within an insulating layer with a very high barrier, when neglecting the energy and momentum dependence of the penetration length. In what follows, we express all energies in units of $\gamma$ for convenience.

To calculate the DOS of the hybrid $N / S$ system [region (II) in Fig. 1] and, later, to study the scattering and transport properties of quasiparticles incoming from normal region (I), we use a volatile numerical method used in studies of the magnetoresistance of hybrid systems, ${ }^{51,52}$ phonon transport, ${ }^{53}$ and more recently in molecular electronics. ${ }^{54}$ It is based on recursive Green function techniques. Although some of the implementation details may differ, ${ }^{55}$ the main stages of the computational scheme are explained in Ref. 52.

\section{B. Quasiparticle density of states}

The proximity effect is reflected in the DOS, $\nu(\epsilon)$, of the hybrid system plotted in Fig. 2 for $\gamma_{N S}=0.32$ and $U_{0}=0$. The solid line corresponds to the semi-infinite geometry shown in Fig. 1(a). In this case $\nu(\epsilon)$ is calculated from the Green function $G\left(i, j, i^{\prime}, j^{\prime} ; \epsilon\right)$ of region (II) via the well-known relation $\nu(\epsilon)=-(1 / \pi N) \operatorname{Im} \sum_{i, j} G(i, j, i, j ; \epsilon)$, where the summation is over all lattice sites in the hybrid part of the junction; the factor $N$ normalizes the area under the curve to a reference unit and Im means the imaginary part. The dashed line corresponds to the geometry of Fig. 1(b) for a relatively long wire $L / \xi_{S} \gg 1$. In this case, we obtain $\nu(\epsilon)$ using a recursive technique (negative-factor counting ${ }^{56}$ ) that allows one to calculate the effective (renormalized) interaction between the normal leads ${ }^{50}$ by projecting out the degrees of freedom of the middle region (II).

Both approaches reveal the formation of a two-gap structure: a smaller gap (minigap) at $E_{g} \approx 0.21 \Delta$ and the usual BCS singularity at $\epsilon=\Delta$ with a finite quasiparticle contribution at intermediate energies, $E_{g}<\epsilon<\Delta$. Figure 3(a) shows 

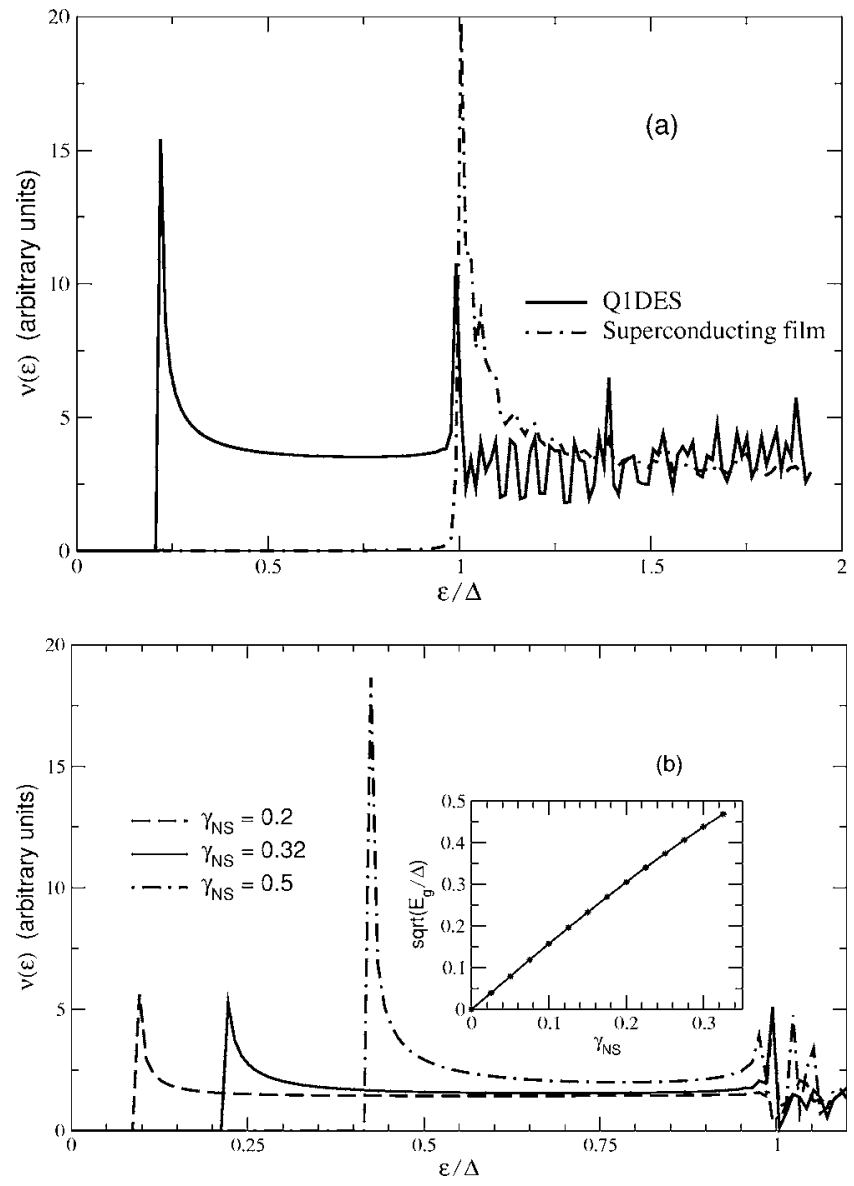

FIG. 3. (a) Decomposition of the DOS $\nu(\epsilon)$ to that arising from the Q1DES (solid curve) and the superconductor (dashed curve). Both curves are scaled to compare with each other. (b) DOS in the proximity region for various interfacial transparencies. Inset: the minigap $E_{g}$ depends quadratically on the interfacial coupling $\gamma_{N S}$ at low transparencies, and starts deviating at higher values of $\gamma_{N S}$.

the DOS separately for the Q1DES (solid line) and for the superconductor (dashed line) from which one can conclude that the smaller gap opens in the DOS of the Q1DES. This observation along with the dependence of $E_{g}$ on the coupling to the superconductor shown in Fig. 3(b) suggests that the minigap formation is a signature of the superconducting correlations induced in the Q1DES. They are maintained in the course of multiple Andreev reflections in the channel which mix particle and hole states with energies below the effective pairing energy coinciding with $E_{g}{ }^{25}$ At higher energies $E_{g}$ $\ll \epsilon<\Delta$ the electrons and holes in the Q1DES are weakly correlated and hence can be treated as one-particle excitations.

In mesoscopically large diffusive $N / S$. systems the formation of the minigap has been studied in a number of theoretical papers (see, e.g, Refs. 40, 41, and 43). In the clean limit, the minigap structure has been analyzed to some extent in billiard geometries resembling quantum dots. ${ }^{45-47}$ Below we develop an analytical model, close in spirit to our numerical approach, that provides a simple description of the superconducting correlations in ballistic wires based on a 1D BdGlike equation with an effective proximity-induced pairing energy $E_{g}$.

\section{Proximity effect in a clean quantum wire: An analytical model}

Although the physical mechanism responsible for the minigap formation in ballistic 2D electron systems has been explored in Ref. 25, the proposed method of derivation of $E_{g}$ heavily relies on the following assumptions. First, the pairing potential in the superconductor $\Delta(z)$ was assumed homogeneous. Secondly, a finite-thickness normal system was modeled by a rectangular confining potential $U_{c}(z)$ enabling a plane wave description of the multiple reflection in the normal channel. The advantage of the model of Ref. 25 is that it allows one to obtain the minigap for an arbitrary interfacial transparency. In this subsection we present an alternative microscopic derivation of $E_{g}$ that does not rely on any particular models for $\Delta(z)$ and $U_{c}(z)$, but is restricted to low interfacial transparencies and low energies $|\epsilon| \sim E_{g} \ll \Delta$. By focusing on this case (weak-coupling regime) we would like to emphasize that the effects related to the minigap formation can be observed even in samples with average interfacial quality provided that the temperature is low enough. As in Ref. 25, we also assume the translational invariance along the $N / S$ interface and different Fermi energies $\mu_{S} \gg \mu_{N}$ and Fermi momenta $p_{S} \gg p_{N}$ on the $S$ and $N$ sides.

It is convenient to rewrite the BdG equation (1) for the two-component wavefunction $\psi_{p}(z)=\left[u_{p}(z), v_{p}(z)\right]^{T}$ in the superconductor $(z \geqslant 0)$ in a more compact form:

$$
\left[\epsilon \sigma_{3}+\left(E_{S}+\frac{\hbar^{2}}{2 m} \partial_{z}^{2}\right) \sigma_{0}-\Delta(z) i \sigma_{2}\right] \psi_{p}(z)=0 .
$$

Here, $p \equiv p_{x}$ is the momentum parallel to the interface; $\sigma_{2,3}$ and $\sigma_{0}$ are the Pauli and unity matrices, respectively. In the normal system $(z \leqslant 0)$ the equation for $\psi_{p}(z)$ is

$$
\left\{\epsilon \sigma_{3}+\left[E_{N}+\frac{\hbar^{2}}{2 m} \partial_{z}^{2}-U_{c}(z)\right] \sigma_{0}\right\} \psi_{p}(z)=0,
$$

with $E_{N, S}=\mu_{N, S}-p^{2} / 2 m$. The confining potential $U_{c}(z)$ defines a Q1D channel with a localized electron wavefunction $\phi(z)$ in the $z$ direction.

The interfacial barrier is assumed rectangular with the electron penetration length $\kappa_{0}^{-1}=\hbar /(2 m U)^{1 / 2}$ determined by the barrier height $U$ measured from the Fermi energy. Inside a high enough barrier one can neglect the energy and momentum dependence of the penetration length and write the BdG equation as $\left[\partial_{z}^{2}-\kappa_{0}^{2}\right] \widetilde{\psi}_{p}(z)=0,0 \leqslant z \leqslant a$, where $a$ is the barrier thickness. We introduce a special notation $\widetilde{\psi}_{p}(z)$ for the $\mathrm{BdG}$ wave function inside the barrier to distinguish it from that outside the barrier. The continuity of the particle current imposes usual boundary conditions at the barrier walls, reading

$$
\begin{gathered}
\tilde{\psi}_{p}(0)=\psi_{p}(0), \quad \tilde{\psi}_{p}(a)=\psi_{p}(a), \\
\partial_{z} \widetilde{\psi}_{p}(0)=\partial_{z} \psi_{p}(0), \quad \partial_{z} \tilde{\psi}_{p}(a)=\partial_{z} \psi_{p}(a) .
\end{gathered}
$$

The solution inside the barrier satisfying the boundary condition (6) is $\tilde{\psi}_{p}(z)=\left[\sinh \kappa_{0}(a-z) / \sinh \kappa_{0} a\right] \psi_{p}(0)$ 
$+\left(\sinh \kappa_{0} z / \sinh \kappa_{0} a\right) \psi_{p}(a)$ Inserting it into the boundary conditions (7) for the derivatives, we have:

$$
\begin{gathered}
\partial_{z} \psi_{p}(0)+\kappa \psi_{p}(0)=\kappa_{t} \psi_{p}(a), \\
\partial_{z} \psi_{p}(a)-\kappa \psi_{p}(a)=-\kappa_{t} \psi_{p}(0),
\end{gathered}
$$

where $\kappa=\kappa_{0} \operatorname{cotanh} \kappa_{0} a$ and $\kappa_{t}=\kappa_{0} / \sinh \kappa_{0} a$. Equations (8) and (9) serve now as effective boundary conditions for the $\mathrm{BdG}$ equations in the superconductor and the normal system. In the limit sinh $\kappa_{0} a \rightarrow \infty$, the coupling between the "normal" and the "superconducting" functions vanishes, which is described by Eqs. (8) and (9) with zero right-hand sides.

We use boundary conditions (8) and (9) to describe Andreev reflection at the superconductor-Q1DES interface under the assumption that the influence of the Q1DES on the superconductor can be neglected. To proceed, it is convenient to include the boundary condition (9) into the BdG equation (4) by introducing appropriate delta-function terms as follows

$$
\begin{aligned}
& {\left[\epsilon \sigma_{3}+\left(E_{S}+\frac{\hbar^{2}}{2 m} \partial_{z}^{2}+\hat{U}_{S}(z)\right) \sigma_{0}-\Delta(z) i \sigma_{2}\right] \psi_{p}(z)} \\
& =-\frac{\kappa_{t} \hbar^{2}}{2 m} \delta(z-a) \psi_{p}(0) .
\end{aligned}
$$

We note that the admitted singular potential $\hat{U}_{S}(z)$ $\equiv\left(\hbar^{2} / 2 m\right) \delta(z-a)\left(\partial_{z}-\kappa\right)$ reproduces Eq. (9) with zero righthand side ("isolated superconductor").

The penetration of Andreev bound states into the superconductor at low energies is described by a particular solution of Eq. (10) generated by the right-hand side containing the "normal" function $\psi_{p}(0)$. It can be expressed in terms of the matrix Green function of Eq. (10) whose matrix elements are constructed from the quasiparticle $G_{p, \epsilon}\left(z, z^{\prime}\right)$ and condensate (Gorkov's) $F_{p, \epsilon}\left(z, z^{\prime}\right)$ Green functions, namely,

$$
\psi_{p}(z)=-\frac{\kappa_{t} \hbar^{2}}{2 m}\left(\begin{array}{cc}
G_{p, \epsilon}(z, a) & -F_{-p,-\epsilon}(z, a) \\
F_{p, \epsilon}(z, a) & G_{-p,-\epsilon}(z, a)
\end{array}\right) \psi_{p}(0) .
$$

Here the Green functions satisfy boundary condition (9) with zero right-hand side. Inserting this solution into the boundary condition (8) at the "normal" side and neglecting both energy and momentum dependence of the Green functions under conditions $|\epsilon| \ll \Delta$ and $p \leqslant p_{N} \ll p_{S}$, one finds

$$
\partial_{z} \psi_{p}(0)+\kappa \psi_{p}(0)=\frac{\kappa_{t}^{2} \hbar^{2}}{2 m} F i \sigma_{2} \psi_{p}(0),
$$

where $F \equiv F_{p=\epsilon=0}(a, a)$ is the condensate Green function taken at the boundary of the superconductor. We have omitted the terms proportional to $G$ since for $|\epsilon| \ll \Delta$ they would only result in a shift of the dispersion.

The right-hand side of the boundary condition (12), which is off diagonal in the particle-hole space, takes into account the conversion of a particle into a hole (and vice versa) due to Andreev reflection, that occurs simultaneously with normal scattering. In a narrow quantum wire, whose thickness is of order of the Fermi wavelength, the anomalous term in the boundary condition (12) gives rise to an effective pairing energy between particles and holes in the wire. Indeed, combining the equation of motion (5) and the boundary condition (12), one can write

$$
\begin{aligned}
& {\left[\epsilon \sigma_{3}+\left(E_{N}+\frac{\hbar^{2} \partial_{z}^{2}}{2 m}-U_{c}(z)+\hat{U}_{N}(z)\right) \sigma_{0}\right] \psi_{p}(z)} \\
& =-\delta(z)\left(\frac{\kappa_{t} \hbar^{2}}{2 m}\right)^{2} F i \sigma_{2} \psi_{p}(z),
\end{aligned}
$$

where the singular potential $\hat{U}_{N}(z) \equiv-\left(\hbar^{2} / 2 m\right) \delta(z)\left(\partial_{z}+\kappa\right)$ is equivalent to the boundary condition (12) with zero righthand side. For a weakly coupled Q1DES, the spatial dependence of the $\mathrm{BdG}$ function $\psi_{p}(z) \approx \psi_{p} \phi(z)$ is almost unaffected by tunneling. Therefore, multiplying Eq. (13) by $\phi(z)$ and integrating over $z$, one obtains the following onedimensional equation:

$$
\begin{gathered}
{\left[\epsilon \sigma_{3}+\left(\frac{p_{F}^{2}-p^{2}}{2 m}\right) \sigma_{0}-E_{g} i \sigma_{2}\right] \psi_{p}=0,} \\
E_{g} \equiv\left[\kappa_{t} \hbar^{2} \phi(0) / 2 m\right]^{2} F .
\end{gathered}
$$

$E_{g} i \sigma_{2}$ plays the role of the effective singlet pairing energy in the wire; $p_{F}$ denotes the Fermi momentum in the Q1DES.

According to Eq. (14), the excitation spectrum in the Q1DES is $\epsilon_{p}^{ \pm}= \pm\left[v_{F}^{2}\left(|p|-p_{F}\right)^{2}+E_{g}^{2}\right]^{1 / 2}$ with the Fermi velocity $v_{F}=p_{F} / m$. It has an energy gap given by Eq. (15) and, hence, the DOS of the normal system displays a BCS-like singularity at $E_{g}$. To estimate $E_{g}$, one can use the condensate Green function of a superconductor with a homogeneous pairing potential $\Delta$ at zero energy and parallel momentum $F$ $\approx W_{S}^{-1} \Sigma_{p_{z}} \Delta /\left[\Delta^{2}+v_{S}^{2}\left(p_{z}-p_{S}\right)^{2}\right]$, where $v_{S}=p_{S} / m$. The integration over $p_{z}$ gives $F \approx 1 / \hbar v_{S}$. The boundary value $\phi(0)$ of the transverse function can be estimated using the unperturbed boundary condition $\phi(0)=-\kappa^{-1} \partial_{z} \phi(0)$, where on the right-hand side one can use the "hard wall" wave function $\phi(z)=\left(2 / W_{N}\right)^{1 / 2} \sin \pi z / W_{N}$, which gives $|\phi(0)|$ $\approx \kappa^{-1}\left(2 / W_{N}\right)^{1 / 2}\left(\pi / W_{N}\right)$. Thus, the effective pairing energy is

$$
E_{g}=\frac{\hbar}{W_{N} p_{S}} \frac{1}{\sinh ^{2} \kappa_{0} a} E_{0},
$$

with $E_{0}=\hbar^{2} \pi^{2} / 2 m W_{N}^{2}$ being the energy of the lowest occupied subband in the quantum well. Equation (16) is equivalent to the one obtained in Ref. 25 for a strong delta-shaped barrier.

Equation (15) for the minigap $E_{g}$ provides a link to the numerical approach and results discussed earlier. According to Eq. (15), the size of the minigap depends on the parameter $\kappa_{t} \hbar^{2} / 2 m$ that characterizes "hopping" between the systems [see Eq. (11)]. This parameter represents a direct analoge of the coupling constant $\gamma_{N S}$ that determines the size of the minigap in the DOS in our numerical study [see Fig. 3(b)]. Since $E_{g}$ is quadratic in $\kappa_{t} \hbar^{2} / 2 m$, the numerical value of the minigap should scale with $\gamma_{N S}$ as

$$
E_{g} \propto \gamma_{N S}^{2},
$$

which can indeed be verified numerically [see inset in Fig. 3(b)]. In the next section we will see that the parabolic de- 


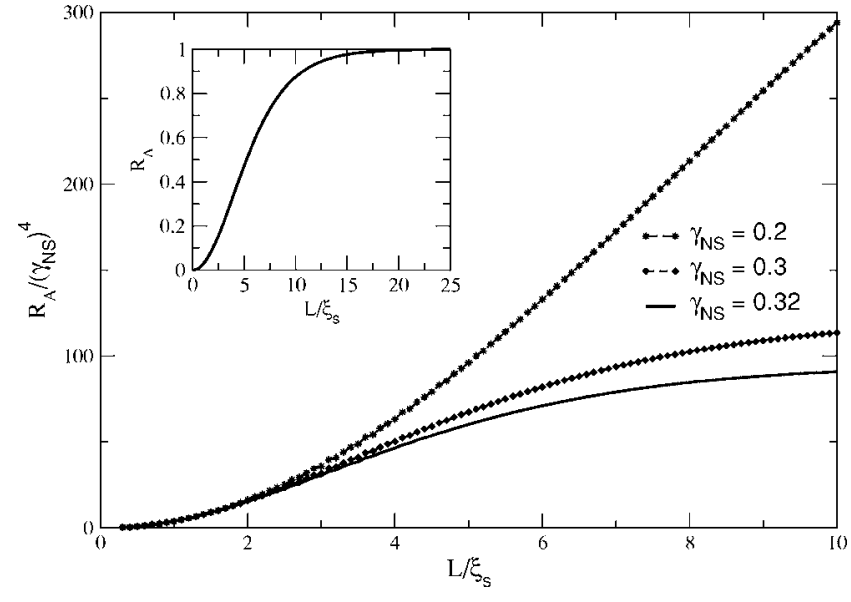

FIG. 4. Andreev reflection probability for various $N / S$ coupling constants as a function of the length of the proximity region (II) in Fig. 2(b). $R_{A}$ scales with the interfacial transparency and at short lengths is quadratic in $L$. For $L$ much larger than the proximityinduced coherence length $\xi_{N} \approx 5 \xi_{S}$, the probability $R_{A}$ reaches unity as a manifestation of the reflectionless tunneling despite the low interfacial transparency (see inset for $\gamma_{N S}=0.2$ ).

pendence of the effective pairing energy (17) on $\gamma_{N S}$ can also be extracted from calculations of the Andreev scattering probability.

\section{QUASIPARTICLE SCATTERING: LENGTH AND ENERGY DEPENDENCE}

In this section we discuss electron scattering properties that can be used as an independent and more complete probe of the proximity effect in finite length parallel $N / S$ contacts. For definiteness we consider particles in the left region (I) of Fig. 1 propagating to the right. When incident at the boundary with the proximity region, these may be (a) Andreev reflected, namely, converted into outgoing holes with the probability $R_{A}$, (b) normally reflected as outgoing particles, i.e., without Andreev conversion, with the probability $R_{N}$, and (c) normally transmitted as particles with the probability $T_{N}$ either in the region (II) of Fig. 1(a) or in the right region (I) of Fig. 1(b). Finally, the probability of being Andreev transmitted to the right is determined via particle conservation, namely, $1-R_{A}-R_{N}-T_{N}$. In our calculations, this is ensured by the unitarity of the scattering matrix.

We examine first the dependence of the zero-energy Andreev reflection coefficient $R_{A}(\epsilon=0)$ on the length $L$ of the proximity region (II) in Fig. 1(b) for different values of the coupling parameter $\gamma_{N S}$ and without any potential mismatch at the $(\mathrm{I}) /(\mathrm{II})$ boundary $\left(U_{0}=0\right)$. In conventional $N / S / N$ structures the Andreev coefficient is known to scale as $R_{A}$ $\sim(\Delta L)^{2}$ for $L$ much shorter than the coherence length $\xi_{S} \cdot{ }^{57}$ According to the results of the previous section, in our case the effective pairing energy $E_{g}$ [Eq. (15)] should act as $\Delta$ and therefore we expect that $R_{A} \sim\left(E_{g} L\right)^{2}$ or, according to Eq. (17), $R_{A} \sim \gamma_{N S}^{4} L^{2}$ for short enough contacts. This scaling is demonstrated in Fig. 4 by the convergence of the appropriately normalized $R_{A}$ curves and their parabolic shape at short lengths. As shown in the inset, there is a characteristic length $\xi_{N} \approx 5 \xi_{S}$ beyond which the Andreev probability $R_{A}(L)$ approaches its unit limit. Moreover, the ratio of $\xi_{N} / \xi_{S}$ coincides with the ratio of the gaps $\Delta / E_{g} \approx 5$ found from the analysis of the DOS in the previous section

$$
\xi_{N} / \xi_{S}=\Delta / E_{g}
$$

The overall length dependence implies that the reflectionless tunneling builds up due to the strong mixing of particles and holes in long channels. In particular, the semiclassical approaches of Refs. 27 and 35 interpret reflectionless tunneling in terms of the increase in the cumulative Andreev reflection probability with increasing number of single Andreev reflections at the $N / S$ boundary in the limiting case of an infinitely long interface $L / \xi_{N} \rightarrow \infty$.

We now turn to the discussion of scattering of finiteenergy quasiparticles in the semi-infinite geometry of the proximity region [Fig. 1(a)] where the reflectionless tunneling is most pronounced. We also take into account a finite potential step $U_{0}$ at the boundary between the normal (I) and proximity (II) regions that, as has been already mentioned, may arise from the modification of the Fermi energy in region (II) due to the coupling to the superconductor. The energy dependence of the Andreev reflection coefficient is plotted in Fig. 5(a) for various $U_{0}$ and $\gamma_{N S}=0.32$. At low energies $\epsilon \leqslant E_{g}=0.21 \Delta$ the shape of the dependence $R_{A}(\epsilon)$ resembles that of high-transparency $N / S$ point contacts discussed by Blonder, Tinkham, and Klapwijk (BTK). ${ }^{21}$ If there is no potential step $U_{0}$ between the normal (I) and proximity (II) regions, the probability $R_{A}$ equals unity and starts to drop at $\epsilon \geqslant E_{g}$. For $U_{0} \neq 0$, finite normal reflection $R_{N}$ builds up [shown in Fig. 6(a)] which results in smaller zero-energy values of $R_{A}$. The appearance of the second narrow peak at $\epsilon=\Delta^{58}$ manifests the crossover from the reflectionless tunneling regime, which involves a two-particle process, to the usual independent electron tunneling through the barrier.

In Fig. 5(b) we demonstrate that the low- and high-energy peaks in the dependence of $R_{A}$ can be independently fitted by the BTK model. ${ }^{21}$ To fit the low-energy behavior we use the formulas of Table II in Ref. 21 with $\Delta_{\mathrm{BTK}}=E_{g}$ and a small barrier parameter $Z=0.278$. For the tunneling peak we use the same formulas with $\Delta_{\mathrm{BTK}}=\Delta$ and the large barrier parameter $Z=28$. In either of the above limiting cases, the fit is almost perfect. To describe the crossover between them, a more general analytical model is needed.

In Fig. 6 all nonvanishing scattering coefficients are plotted for (a) $U_{0}=0.8$ and (b) no barrier between the normal (I) and proximity (II) regions. For $U_{0} \neq 0$ there is normal reflection of particles at the (I)/(II) interface caused by the potential mismatch. For $U_{0}=0$ it vanishes not only below the minigap $\epsilon<E_{g}$ but also at the intermediate energies $E_{g}<\epsilon<\Delta$. This is due to the specific geometry of our tunnel junction where quasiparticles with intermediate energies are mainly transmitted through the channel experiencing low-probability Andreev reflection [see the behavior of $T_{N}(\epsilon)$ and $R_{A}(\epsilon)$ ]. At higher energies $\epsilon>\Delta$, when the superconductor becomes transparent for quasiparticles, the lack of the translational invariance of our system causes considerable normal scatter- 

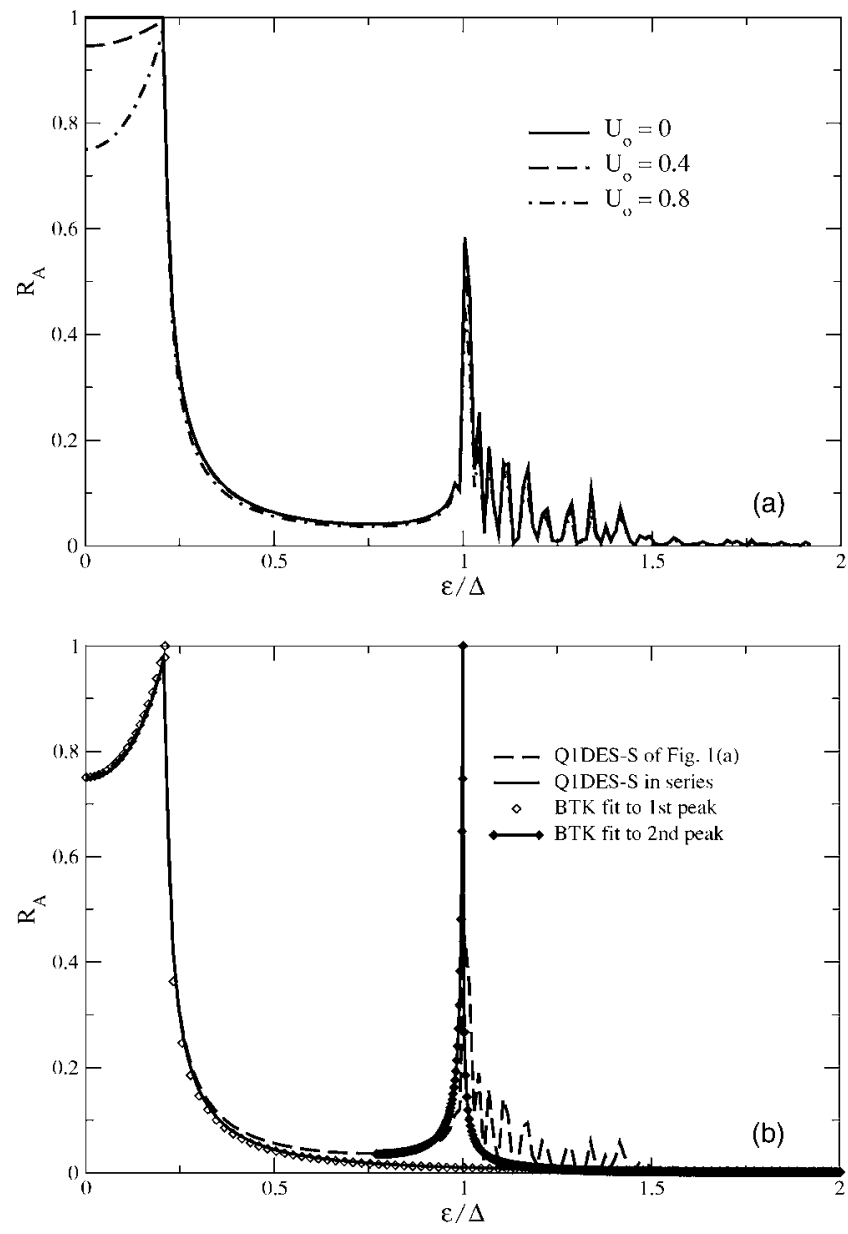

FIG. 5. (a) Andreev reflection coefficient for the geometry of Fig. 1(a) with $\gamma_{N S}=0.32$ and various values of the potential $U_{0}$ at (I)/(II) boundary. For $U_{0}=0$ the Andreev probability is exactly 1 for energies below $E_{g}$. (b) To fit the low- and high-energy peaks we use the formulas of the BTK model (Ref. 21) with parameters $\Delta_{\mathrm{BTK}}$ $=E_{g}, Z=0.278$, and $\Delta_{\mathrm{BTK}}=\Delta, Z=28$, respectively.

ing and oscillations of all the coefficients due to the finite thickness of the superconductor.

\section{CONCLUDING REMARKS}

Scattering coefficients are related to the two-, three-, and in general multiprobe conductances of $N / S$ systems (see, e.g., Refs. 21 and 33). Therefore, the proximity effect discussed in the previous section should be observable in measurements of the current-voltage characteristics of such hybrids. In order to explore this possibility and to elaborate on our discussion of the experimental reports, ${ }^{15,22-24}$ we conclude by focusing on the two-probe differential conductance $g(e V) \equiv d I / d V$ of the semi-infinite geometry of Fig. 1(a). This is given by ${ }^{33}$

$g(e V)=\frac{2 e^{2}}{h} \int_{0}^{\infty} d \epsilon\left\{-\frac{\partial f^{p}}{\partial \epsilon}\left(1-R_{N}^{p}+R_{A}^{p}\right)-\frac{\partial f^{h}}{\partial \epsilon}\left(1-R_{N}^{h}+R_{A}^{h}\right)\right\}$,

where $f^{p(h)}=\left\{\exp \left[(\epsilon \mp e V) / k_{B} T\right]+1\right\}^{-1}$ with (-) for particles
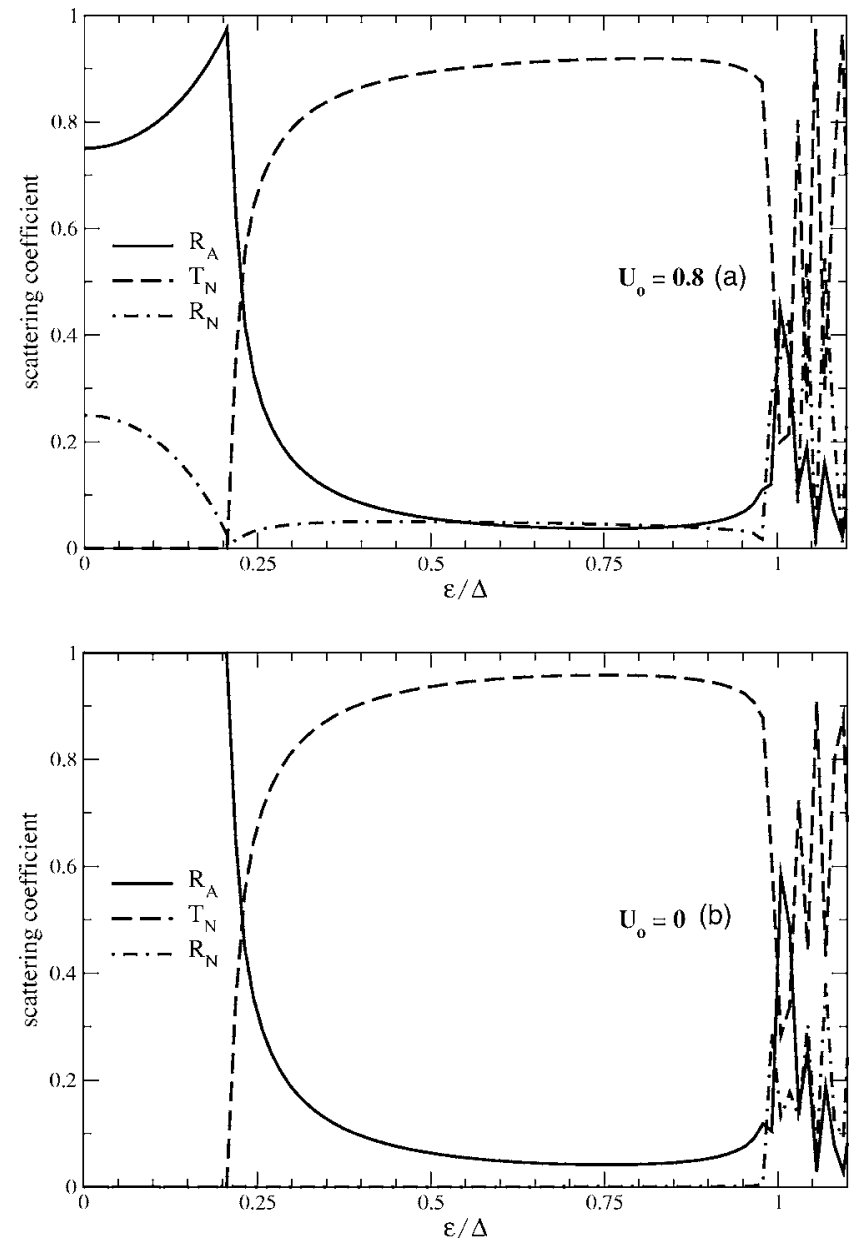

FIG. 6. Andreev reflection $R_{A}$, normal transmission $T_{N}$, and normal reflection $R_{N}$ coefficients for the geometry of Fig. 1(a) with $\gamma_{N S}=0.32$ and various potential landscapes in region (II).

$(p)$ and $(+)$ for holes $(h)$. The bias energy $e V$ is introduced as the difference between the chemical potentials in the normal region and in the hybrid part of the junction, with the latter taken as reference.

At zero temperature, Eq. (19) reduces to $g(e V)=\left(2 e^{2} / h\right)$ $\times\left[1+R_{A}^{p}(e V)-R_{N}^{p}(e V)\right]=\left(2 e^{2} / h\right) \times\left[1+R_{A}^{h}(-e V)-R_{N}^{h}(-e V)\right]$. Hence, for a small barrier between the normal (I) and proximity (II) regions of the wire, the dependence of $g(\mathrm{eV})$ at ultralow $T$ reflects mainly the energy dependence of the Andreev probability [Fig. 5(a)]. The same is true for the length dependence of the zero-bias conductance. In Fig. 7 we plot the differential conductance for several values of interfacial coupling $\gamma_{N S}$ (i.e., $E_{g}$ ) at $T=0.01 \Delta / k_{B}$. Unlike the tunneling peaks at $\pm \Delta / e$, the proximity-induced anomalies at the minigap energy $\pm E_{g} / e$ exhibit a strong dependence on $\gamma_{N S}$ [see Fig. 3(b)].

In Fig. 8, the evolution of the differential resistance, which is defined as the inverse of Eq. (19), is shown as a function of temperature. At intermediate $T \sim E_{g} / k_{B}$, features at the scale of the minigap are smeared and the resistance exhibits an overall dip as a result of the reflectionless tunneling. With decreasing the temperature to $T \ll E_{g} / k_{B}$ the resistance curve develops a finer structure reflecting the energy dependence of the Andreev reflection probability. For vanish- 


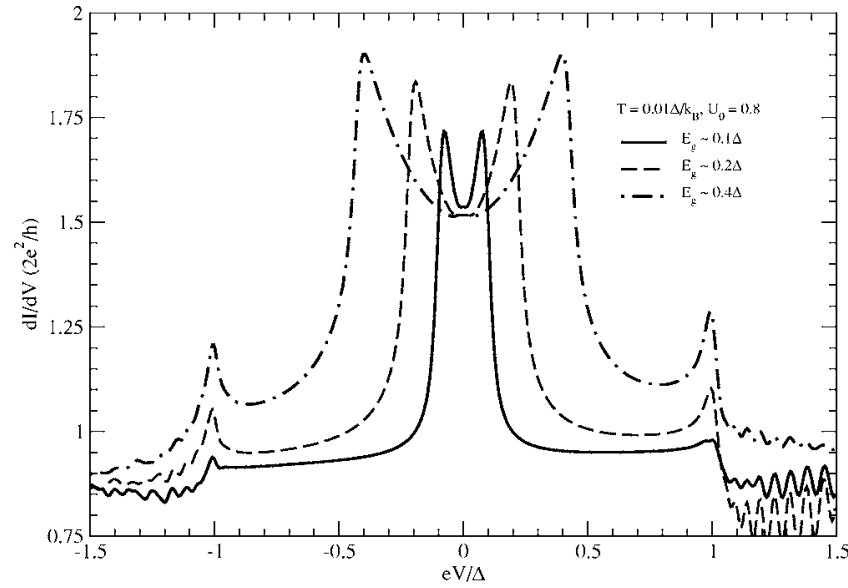

FIG. 7. Differential conductance of the $N / S$ hybrid in Fig. 5(a) at ultralow temperature $T \ll E_{g} / k_{B}$.

ing potential step $U_{0}$ [Fig. 8(a)], there are two minima symmetric to zero bias at the energies of the superconducting gap. In addition to those, for $U_{0} \neq 0$ [Fig. 8(b)] the finite normal reflection at $|\epsilon|<E_{g}$ leads to a zero-bias resistance peak superimposed on the Andreev dip.
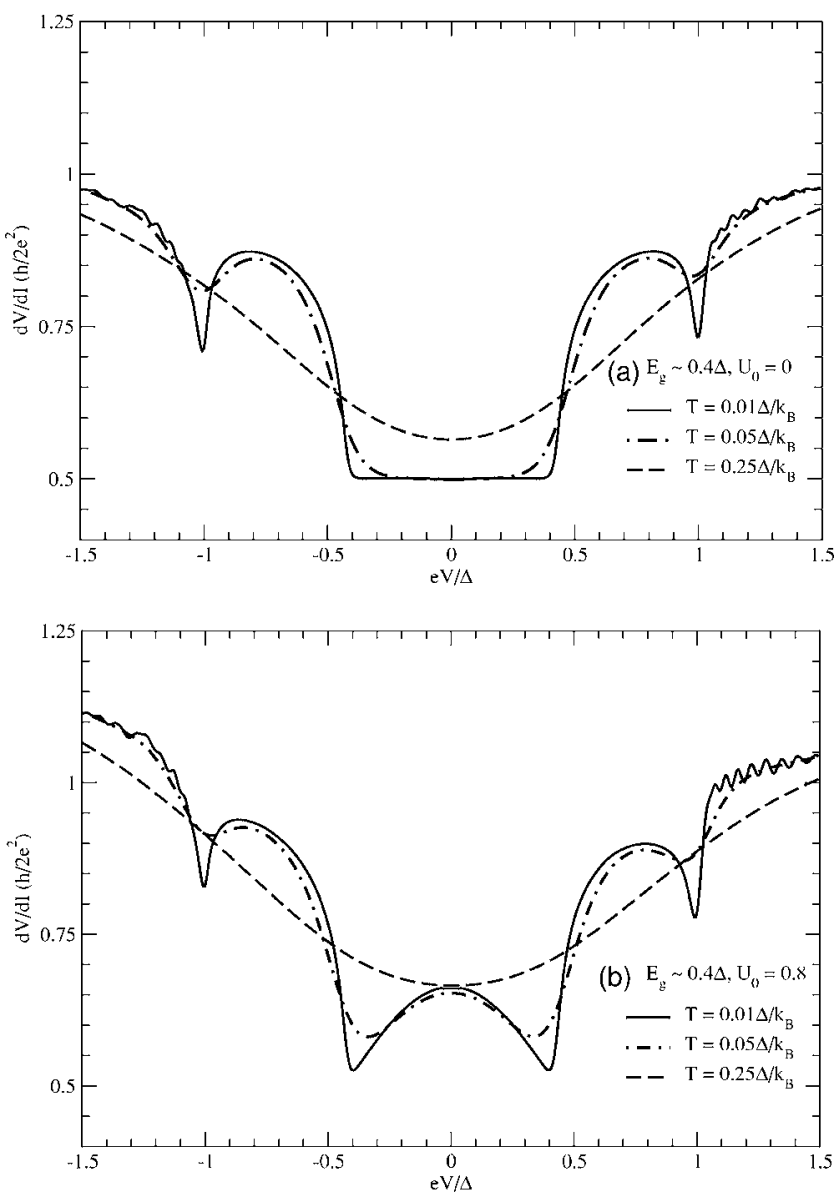

FIG. 8. Evolution of the differential resistance of the $N / S$ hybrid in Fig. 5(a), as a function of temperature from $k_{B} T \sim E_{g}<\Delta$ to $T$ $\ll E_{g}$ for (a) vanishing and (b) finite potential step between regions (I) and (II).
According to Fig. 4, for pronounced Andreev reflection the contact length $L$ must be of order of the proximityinduced coherence length $\xi_{N}=5 \xi_{S}$. For $0.01 \mu \mathrm{m}<\xi_{S}$ $<0.04 \mu \mathrm{m}$ typical for $\mathrm{Nb}$ electrodes, $\xi_{N}$ can be estimated as $0.05 \mu \mathrm{m}<\xi_{N}<0.2 \mu \mathrm{m}$. On the other hand, in the device of Ref. 15 the contact overlap between the nanotube and the superconductor was rather large, about $1 \mu \mathrm{m}$. Therefore, the condition $L>\xi_{N}$ could be met leading to the observed zerobias reduction of the contact resistance. Another feature of our $d V / d I(V)$ curves, namely, the appearance of a small zero-bias peak superimposed on the Andreev dip due to finite normal scattering at very low $T$ [Fig. 8(b)], is also consistent with the experimental findings.

We note that in the experiment of Ref. 15 the low-bias behavior of the resistance was sensitive to a gate voltage applied to the carbon nanotube. In our model the effect of the gate voltage can be incorporated into the difference $U_{0}$ between the Fermi energies in the normal (I) and proximity (II) regions in Fig. 1. We have focused on the most interesting case of relatively small $U_{0}$ when normal scattering does not impede the conversion of the quasiparticle current into the supercurrent in the proximity region. As $U_{0}$ increases, the low-bias resistance dip in Fig. 8(b) eventually evolves into an overall peak above the normal state value as in nonideal $N / S$ point contacts. ${ }^{21,59}$

We emphasize that the zero-bias anomaly discussed here is a property of a single parallel $N / S$ contact. Although as argued in Ref. 15 in their $S / \mathrm{CN} / S$ devices the two $\mathrm{CN} / S$ interfaces acted independently, the role of the interelectrode coupling remains unclear. Such a question has been investigated numerically in Ref. 5 for a somewhat simpler system where a carbon nanotube is connected to a normal metal and a superconductor via tunnel barriers $(N / \mathrm{CN} / S)$. It was shown that resonant tunneling through Andreev levels in the nanotube can significantly increase the low-bias subgap conductance similar to the situation in mesoscopic $N /$ quantum $\operatorname{dot} / S$ structures ${ }^{29}$ For a comprehensive theory of transport in $S / \mathrm{CN} / S$ hybrids this aspect, together with the contact geometry and electron interaction effects, must be taken into account. In addition, one should bear in mind that in the experimental realizations a number of CNs have been contacted in parallel.

In Refs. 22 and 24 a strong zero-bias suppression of the resistance was found in ballistic $2 \mathrm{D}$ electron systems in extended planar coupling to superconductors at $T<\Delta / k_{B}$. These systems can be considered as a generalization of that shown in Fig. 1. For perfect planar interfaces, individual channels with possibly different barriers and interfacial transparencies ${ }^{59}$ add up independently. However, interchannel mixing must be considered for rough surfaces. The same applies when considering experiments in quantum wires ${ }^{23}$ with few propagating modes. It is worth noting that in this case the behavior similar to the low- $T$ differential conductance of Fig. 7 was observed. We believe that the transport anomalies observed in $1 \mathrm{D},{ }^{15}$ quasi-1D, ${ }^{23}$ and $2 \mathrm{D}$ (Refs. 22 and 24) systems have a contribution of a common nature stemming from the proximity-induced mixing of particles and holes which mediate the conversion of a normal current into a supercurrent along the contact on the scale of the coherence length $\xi_{N}=\xi_{S} \Delta / E_{g}$ and at energies smaller than the minigap $E_{g}<\Delta$. 


\section{ACKNOWLEDGMENTS}

We thank A. F. Morpurgo for communicating the geometrical parameters of $S / C N / S$ structures studied in Ref. 15 . G.F. acknowledges funding by the ATOM CAD project within the Sixth Framework Programme of the EU and by the Science Foundation Ireland. This work was also supported by the Deutsche Forschungsgemeinschaft (Forschergruppe 370, Graduiertenkolleg 638).
*Electronic address: gfagas@tyndall.ie

$\dagger$ Electronic address: Grigory.Tkachov@physik.uni-r.de

${ }^{1}$ R. Fazio, F. W. J. Hekking, and A. A. Odintsov, Phys. Rev. Lett. 74, 1843 (1995); C. Winkelholz, R. Fazio, F. W. J. Hekking, and G. Schön, ibid. 77, 3200 (1996).

${ }^{2}$ D. L. Maslov, M. Stone, P. M. Goldbart, and D. Loss, Phys. Rev. B 53, 1548 (1996).

${ }^{3}$ Y. Takane, J. Phys. Soc. Jpn. 66, 537 (1997).

${ }^{4}$ I. Affleck, J.-S. Caux, and A. M. Zagoskin, Phys. Rev. B 62, 1433 (2000).

${ }^{5}$ Y. Wei, J. Wang, H. Guo, H. Mehrez, and C. Roland, Phys. Rev. B 63, 195412 (2001).

${ }^{6}$ M. Titov, N. A. Mortensen, H. Schomerus, and C. W. J. Beenakker, Phys. Rev. B 64, 134206 (2001).

${ }^{7}$ J. Gonzalez, Phys. Rev. Lett. 87, 136401 (2001); J. Phys.: Condens. Matter 15, S2473 (2003).

${ }^{8}$ S. Vishveshwara, C. Bena, L. Balents, and M. P. A. Fisher, Phys. Rev. B 66, 165411 (2002).

${ }^{9}$ B. K. Nikolic, J. K. Freericks, and P. Miller, Phys. Rev. Lett. 88, 077002 (2002).

${ }^{10}$ H.-W. Lee, Hyun C. Lee, Hangmo Yi, and Han-Yong Choi, Phys. Rev. Lett. 90, 247001 (2003).

${ }^{11}$ J. Jiang, L. Yang, J. Dong, and D. Y. Xing, Phys. Rev. B 68, 054519 (2003).

${ }^{12}$ E. Vecino, A. Martín-Rodero, and A. L. Yeyati, Phys. Rev. B 68, 035105 (2003).

${ }^{13}$ Y. Tanaka, A. A. Golubov, and S. Kashiwaya, Phys. Rev. B 68, 054513 (2003).

${ }^{14}$ A. Yu. Kasumov, R. DeBlock, M. Kociak, B. Reulet, H. Bouchiat, I. I. Khodos, Yu. B. Gorbatov, V. T. Volkov, C. Journet, and M. Burghard, Science 84, 1508 (1999); A. Kasumov, M. Kociak, M. Ferrier, R. Deblock, S. Gueron, B. Reulet, I. Khodos, O. Stephan, and H. Bouchiat, Phys. Rev. B 68, 214521 (2003).

${ }^{15}$ A. F. Morpurgo, J. Kong, C. M. Marcus, and H. Dai, Science 286, 263 (1999); Physica B 280, 382 (2000).

${ }^{16}$ M. R. Buitelaar, T. Nussbaumer, and C. Schönenberger, Phys. Rev. Lett. 89, 256801 (2002).

${ }^{17}$ J. Haruyama, K. Takazawa, S. Miyadai, A. Takeda, N. Hori, I. Takesue, Y. Kanda, N. Sugiyama, T. Akazaki, and H. Takayanagi, Phys. Rev. B 68, 165420 (2003); J. Haruyama, A. Tokita, N. Kobayashi, M. Nomura, S. Miyadai, K. Takazawa, A. Takeda, and Y. Kanda, Appl. Phys. Lett. 84, 4714 (2004).

${ }^{18}$ M. Bockrath, D. H. Cobden, J. Lu, A. G. Rinzler, R. E. Smalley, L. Balents, and P. L. McEuen, Nature (London) 397, 598 (1999).

${ }^{19}$ M. S. Dresselhaus, G. Dresselhaus, and P. C. Eklund, Science of Fullerenes and Carbon Nanotubes (Academic, San Diego, 1996).

${ }^{20}$ A. F. Andreev, Zh. Eksp. Teor. Fiz. 46, 1823 (1964) [Sov. Phys.
JETP 19, 1228 (1964)].

${ }^{21}$ G. E. Blonder, M. Tinkham, and T. M. Klapwijk, Phys. Rev. B 25, 4515 (1982).

${ }^{22}$ C. Nguyen, H. Kroemer, and E. L. Hu, Phys. Rev. Lett. 69, 2847 (1992).

${ }^{23}$ F. Rahman and T. J. Thornton, Superlattices Microstruct. 25, 767 (1999).

${ }^{24}$ J. Eroms, M. Tolkiehn, D. Weiss, U. Rössler, J. DeBoeck, and S. Borghs, Europhys. Lett. 58, 569 (2002); J. Eroms, Ph.D. thesis, Universität Regensburg, Regensburg, 2002.

${ }^{25}$ A. F. Volkov, P. H. C. Magnee, B. J. van Wees, and T. M. Klapwijk, Physica C 242, 261 (1995).

${ }^{26}$ A. Kastalsky, A. W. Kleinsasser, L. H. Greene, R. Bhat, F. P. Milliken, and J. P. Harbison, Phys. Rev. Lett. 67, 3026 (1991).

${ }^{27}$ B. J. van Wees, P. de Vries, P. Magnee, and T. M. Klapwijk, Phys. Rev. Lett. 69, 510 (1992).

${ }^{28}$ A. F. Volkov, Phys. Lett. A 174, 144 (1993); A. F. Volkov, A. V. Zaitsev, and T. M. Klapwijk, Physica C 210, 21 (1993).

${ }^{29}$ C. W. J. Beenakker, Phys. Rev. B 46, 12841 (1992); Rev. Mod. Phys. 69, 731 (1997).

${ }^{30}$ I. K. Marmorkos, C. W. J. Beenakker, and R. A. Jalabert, Phys. Rev. B 48, 2811 (1993).

${ }^{31}$ J. Nitta, T. Akazaki, and H. Takayanagi, Phys. Rev. B 49, 3659 (1994).

${ }^{32}$ W. Poirier, D. Mailly, and M. Sanquer, Phys. Rev. Lett. 79, 2105 (1997).

${ }^{33}$ C. J. Lambert and R. Raimondi, J. Phys.: Condens. Matter 10, 901 (1998).

${ }^{34}$ G. B. Lesovik, A. L. Fauchere, and G. Blatter, Phys. Rev. B 55, 3146 (1997).

${ }^{35}$ M. Schechter, Y. Imry, and Y. Levinson, Phys. Rev. B 64, 224513 (2001).

${ }^{36}$ I. E. Batov, Th. Schäpers, A. A. Golubov, and A. V. Ustinov, J. Appl. Phys. 96, 3366 (2004).

${ }^{37}$ D. Ferry and S. M. Goodnick, Transport in Nanostructures (Cambridge University Press, Cambridge, 2000).

${ }^{38}$ V. T. Petrashov, V. N. Antonov, P. Delsing, and T. Claeson, Phys. Rev. Lett. 74, 5268 (1995); W. Belzig, R. Shaikhaidarov, V. V. Petrashov, and Yu. V. Nazarov, Phys. Rev. B 66, 220505(R) (2002).

${ }^{39}$ H. Courtois, Ph. Gandit, D. Mailly, and B. Pannetier, Phys. Rev. Lett. 76, 130 (1996); P. Dubos, H. Courtois, O. Buisson, and B. Pannetier, ibid. 87, 206801 (2001).

${ }^{40}$ A. A. Golubov, E. P. Houwman, J. G. Gijsbertsen, V. M. Krasnov, J. Flokstra, H. Rogalla, and M. Yu. Kupriyanov, Phys. Rev. B 51, 1073 (1995); B. A. Aminov, A. A. Golubov, and M. Yu. Kupriyanov, ibid. 53, 365 (1996).

${ }^{41}$ W. Belzig, C. Bruder, and G. Schön, Phys. Rev. B 54, 9443 (1996).

${ }^{42}$ A. Kadigrobov, L. Y. Gorelik, R. I. Shekhter, M. Jonson, R. Sh. 
Shaikhaidarov, V. T. Petrashov, P. Delsing, and T. Claeson, Phys. Rev. B 60, 14589 (1999).

${ }^{43}$ A. Altland, B. D. Simons, and D. Taras-Semchuk, Adv. Phys. 49, 321 (2000).

${ }^{44}$ A. Anthore, H. Pothier, and D. Esteve, Phys. Rev. Lett. 90, 127001 (2003).

${ }^{45}$ J. A. Melsen, P. W. Brouwer, K. M. Frahm, and C. W. J. Beenakker, Europhys. Lett. 35, 7 (1996); H. Schomerus and C. W. J. Beenakker, Phys. Rev. Lett. 82, 2951 (1999); P. Jacquod, H. Schomerus, and C. W. J. Beenakker, ibid. 90, 207004 (2003).

${ }^{46}$ W. Ihra, M. Leadbeater, J. L. Vega, and K. Richter, Eur. Phys. J. B 21, 425 (2001).

${ }^{47}$ J. Cserti, A. Kormányos, Z. Kaufmann, J. Koltai, and C. J. Lambert, Phys. Rev. Lett. 89, 057001 (2002); J. Cserti, P. Polinak, G. Palla, U. Zülicke, and C. J. Lambert, Phys. Rev. B 69, 134514 (2004).

${ }^{48}$ R. A. Morrow and K. R. Brownstein, Phys. Rev. B 30, 678 (1984).

${ }^{49}$ Although $\xi_{S} / \lambda_{F} \gg 1$ in real superconducting materials, the actual value of this ratio does not affect our results other than increasing significantly the computational cost if made much greater than unity.

${ }^{50}$ P-O. Löwdin, J. Math. Phys. 3, 969 (1962).

${ }^{51}$ F. Taddei, S. Sanvito, and C. J. Lambert, Phys. Rev. B 63,
012404 (2001).

${ }^{52}$ S. Sanvito, C. J. Lambert, J. H. Jefferson, and A. M. Bratkovsky, Phys. Rev. B 59, 11936 (1999).

${ }^{53}$ G. Fagas, A. G. Kozorezov, C. J. Lambert, J. K. Wigmore, A. Peacock, A. Poelaert, and R. denHartog et al., Phys. Rev. B 60, 6459 (1999).

${ }^{54}$ G. Fagas, A. Kambili, and M. Elstner, Chem. Phys. Lett. 389, 268 (2004).

${ }^{55}$ We use an optimised F90 implementation developed by one of the authors (G.F.). It is generalized to include manipulation of nonorthogonal matrices, needed to tackle arbitrary geometries, and of possible non-orthogonal basis sets for applications to materials

${ }^{56}$ M. Leadbeater, Ph.D. thesis, Lancaster University, 1996.

${ }^{57}$ N. R. Claughton, V. C. Hui, and C. J. Lambert, Phys. Rev. B 51, 11635 (1995).

${ }^{58}$ We have checked that $R_{A}(\epsilon)$ approaches unity at both the minigap (when $U_{0} \neq 0$ ) and superconducting gap energies for selected cases. This requires a fine choice of the energy grid due to the singular behavior of the peaks and, hence, increased computational time. Since our results do not depend crucially on this, this practice has been avoided.

${ }^{59}$ N. A. Mortensen, K. Flensberg, and A.-P. Jauho, Phys. Rev. B 59, 10176 (1999). 